Supporting Information

\title{
Synthesis and properties of poly-ether/ethylene carbonate electrolytes with high oxidative stability
}

Kasim $\operatorname{Khan}^{\dagger}$, Zhengyuan Tu ${ }^{\ddagger}$, Qing Zhao ${ }^{\dagger}$, Chenzi Zhao ${ }^{\dagger}$, Lynden A. Archer ${ }^{*}, \ddagger$

${ }^{\dagger}$ Robert Frederick Smith School of Chemical and Biomolecular Engineering, Cornell University, Ithaca, NY, USA.

${ }^{\ddagger}$ Department of Materials Science and Engineering, Cornell University, USA.

\section{Supporting Figures and Tables}

Figure S1: Time-dependent FTIR analysis for a 50\% DOL-50\% EC_1 M LiPF 6 electrolyte.

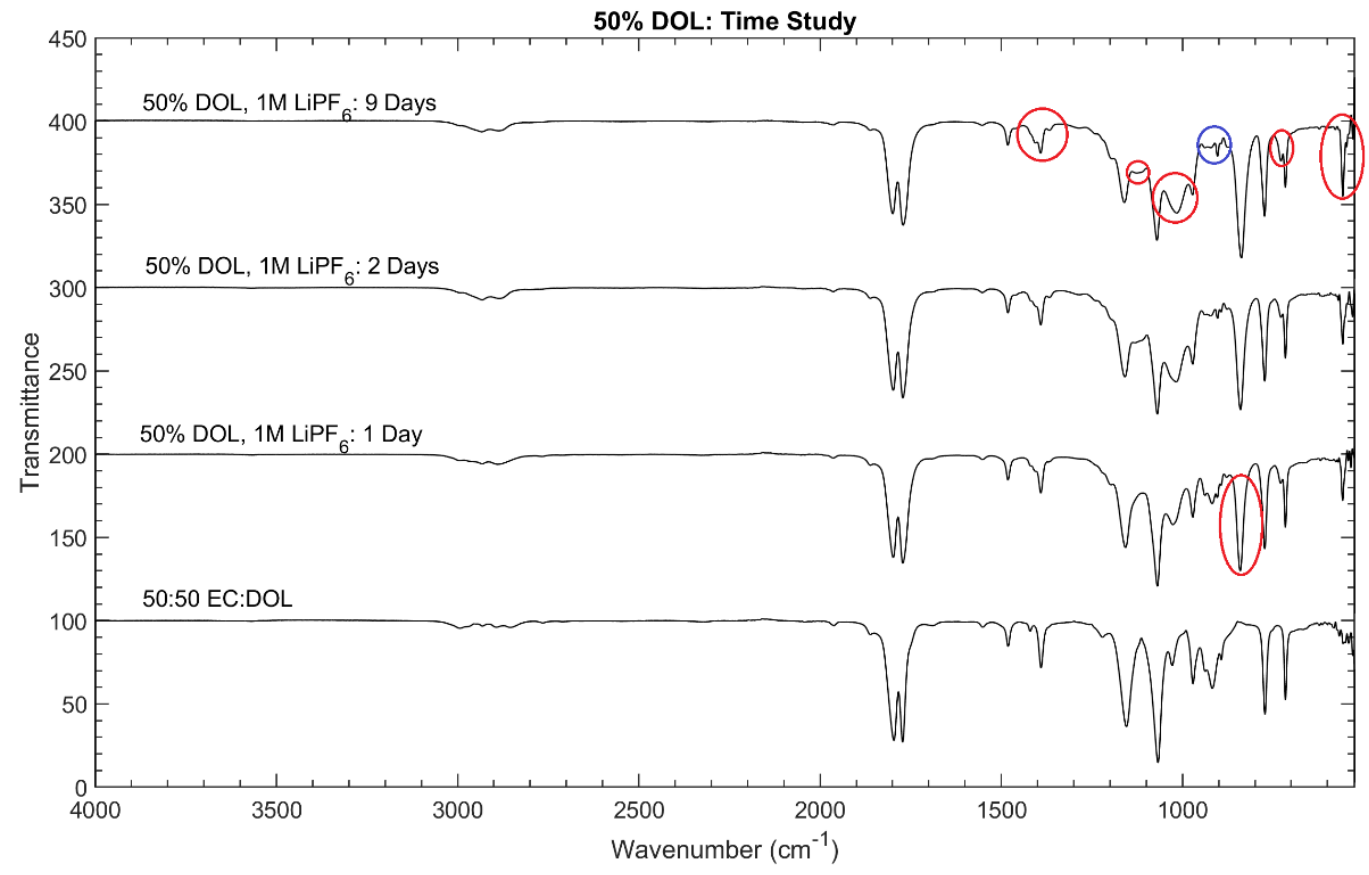


Figure S2: FTIR of $10 \%$ DOL, 90\% EC, $1 \mathrm{M} \mathrm{LiPF}_{6}$

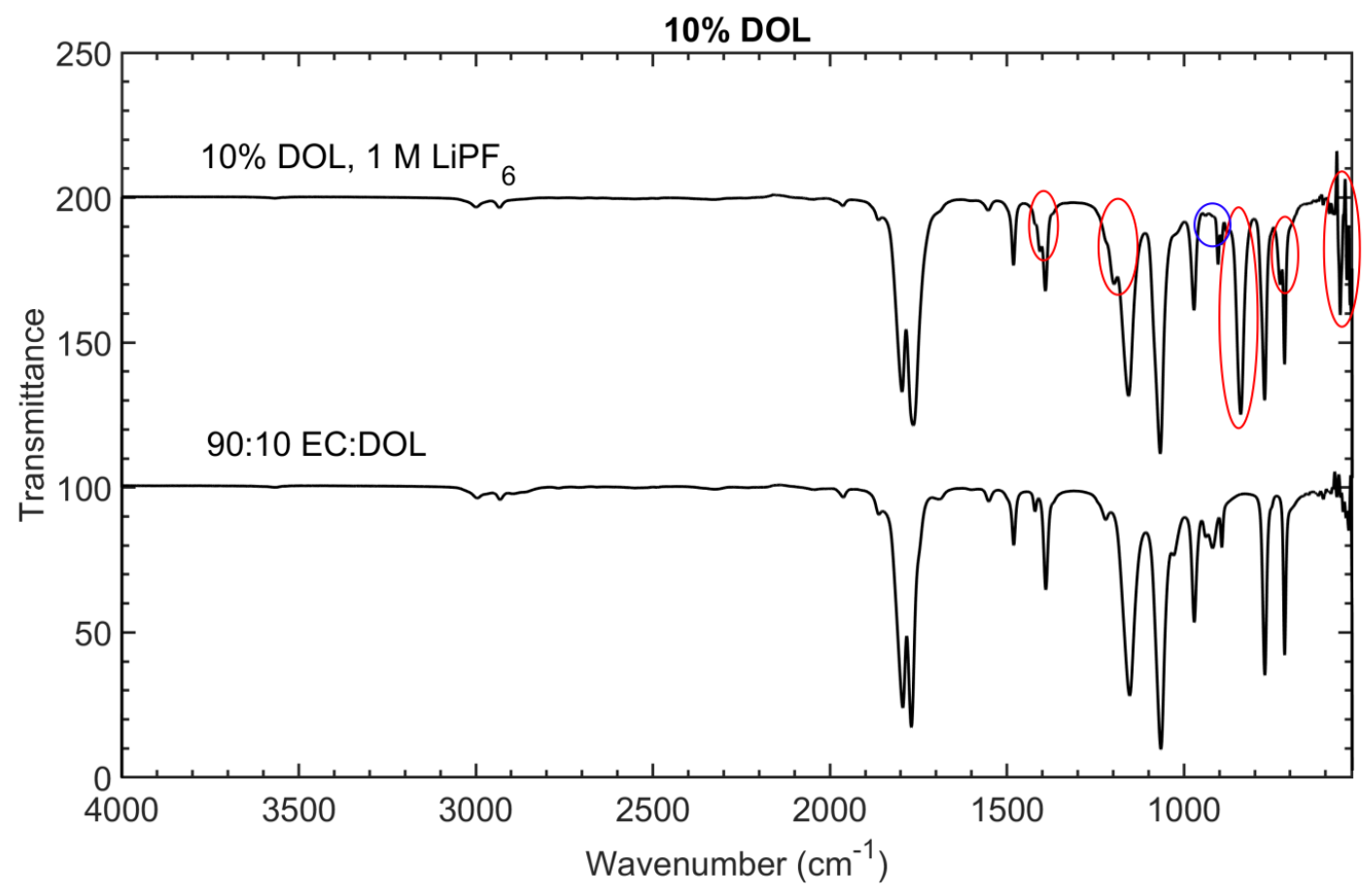

Figure S3: FTIR of DOL, $1 \mathrm{M} \mathrm{LiPF}_{6}$ over time.

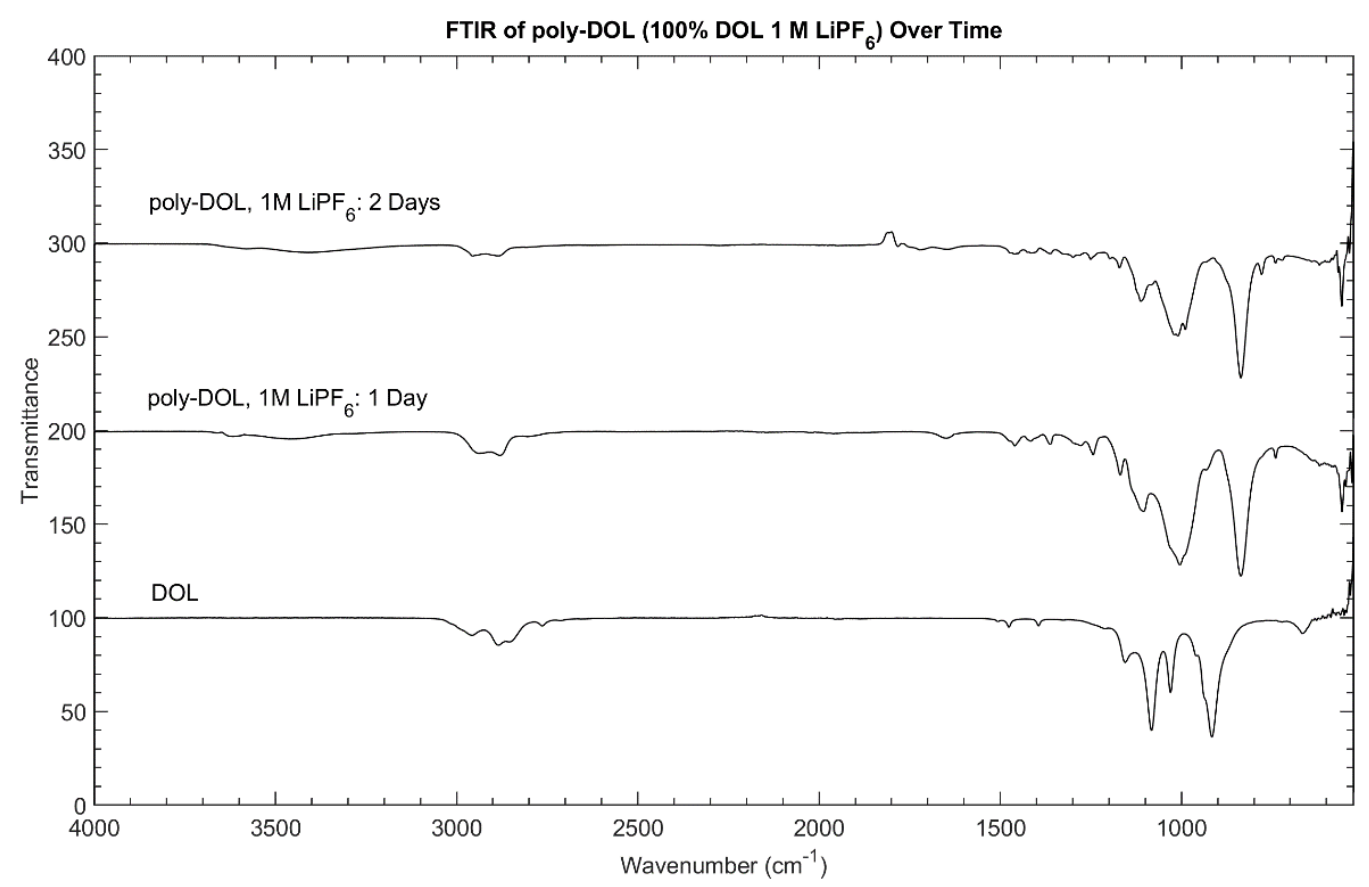


Figure S4: FTIR of poly-DOL degradation (before/after heating to $100{ }^{\circ} \mathrm{C}$ ).

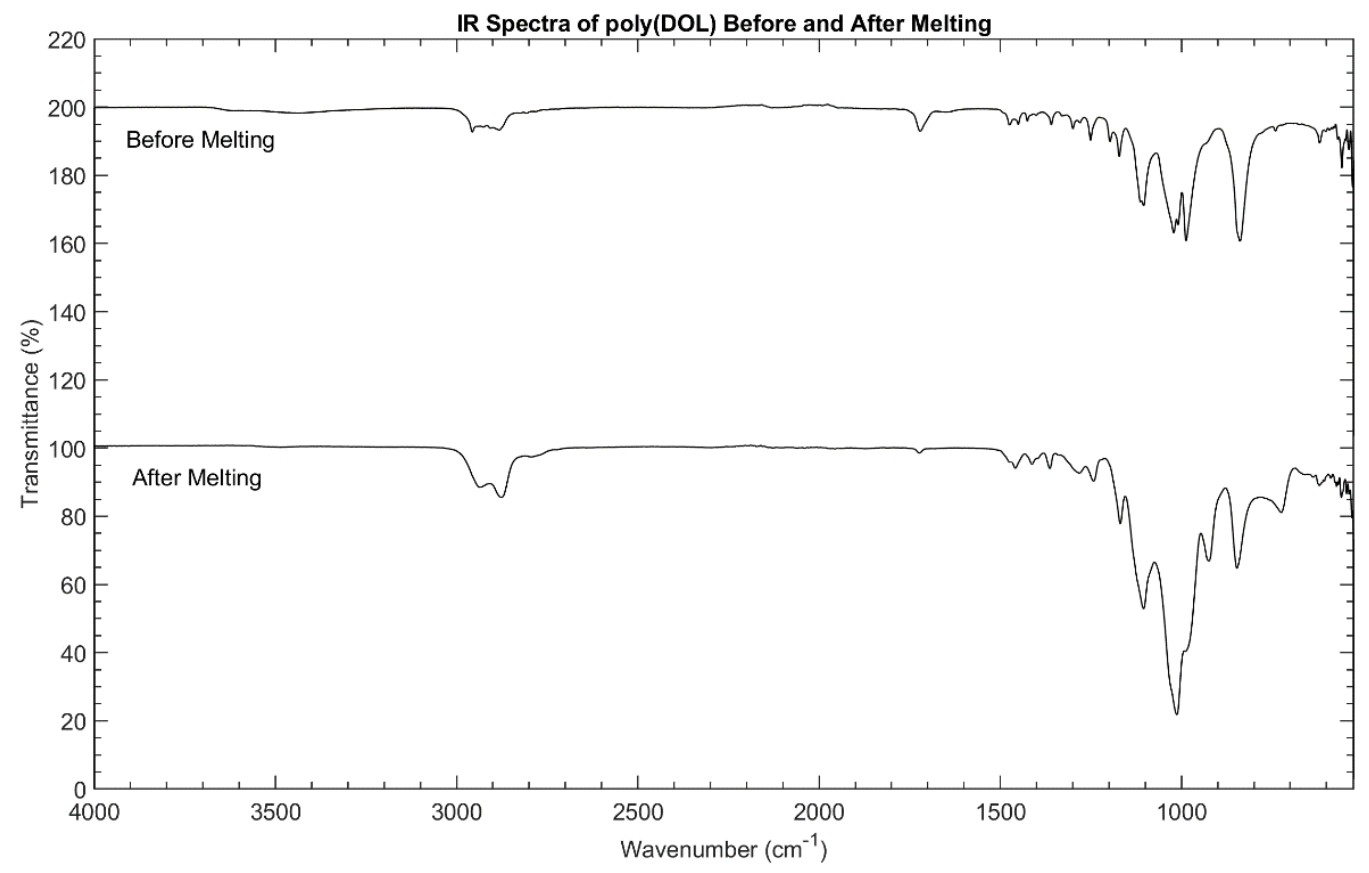

Table S1: Arrhenius fitting data for conductivity with $95 \%$ confidence bounds and $\mathrm{R}^{2}$ values.

Arrhenius fit: $\sigma=A e^{-\frac{E_{a}}{R T}}$

\begin{tabular}{|r|r|r|r|r|r|r|r|r|}
\hline \% DOL & Ea $(\mathbf{e V})$ & Ea $\min (\mathbf{e V})$ & Ea max (eV) & A & A min & A max & $\mathbf{R}^{\wedge} \mathbf{2}$ & Adj. $\mathbf{R}^{\wedge 2}$ \\
\hline $\mathbf{1 0 \%}$ & 0.168 & 0.186 & 0.150 & 0.985 & 0.520 & 1.868 & 0.994 & 0.992 \\
\hline $\mathbf{3 0 \%}$ & 0.172 & 0.183 & 0.161 & 0.897 & 0.611 & 1.315 & 0.998 & 0.997 \\
\hline
\end{tabular}

Table S2: Vogel-Fulcher-Tammann (VFT) fitting data for conductivity with $95 \%$ confidence bounds and $\mathrm{R}^{2}$ values.

VFT fit: $\sigma=A e^{\frac{B}{\left(T-T_{0}\right)}}$

\begin{tabular}{|r|l|r|r|r|r|r|r|r|r|}
\hline \% DOL & $\mathbf{B}(\mathbf{K})$ & $\mathbf{B} \min (\mathbf{K})$ & $\mathbf{B} \max (\mathbf{K})$ & $\mathbf{A}$ & $\mathbf{A} \min$ & $\mathbf{A} \max$ & T0 (=Tg from DSC, K) & $\mathbf{R}^{\wedge 2}$ & Adj. $\mathbf{R}^{\wedge} \mathbf{2}$ \\
\hline $\mathbf{5 0 \%}$ & -516.4 & -544.7 & -488 & 0.048 & 0.037 & 0.062 & 191.65 & 0.998 & 0.997 \\
\hline $\mathbf{7 0 \%}$ & -745.4 & -794.6 & -696.2 & 0.108 & 0.074 & 0.158 & 177.03 & 0.996 & 0.995 \\
\hline
\end{tabular}


Figure S5: Room temperature $\left(25^{\circ} \mathrm{C}\right)$ DC ionic conductivity vs. DOL fraction with a crude logistic fit. Viscosity vs. DOL fraction yields a similar plot.

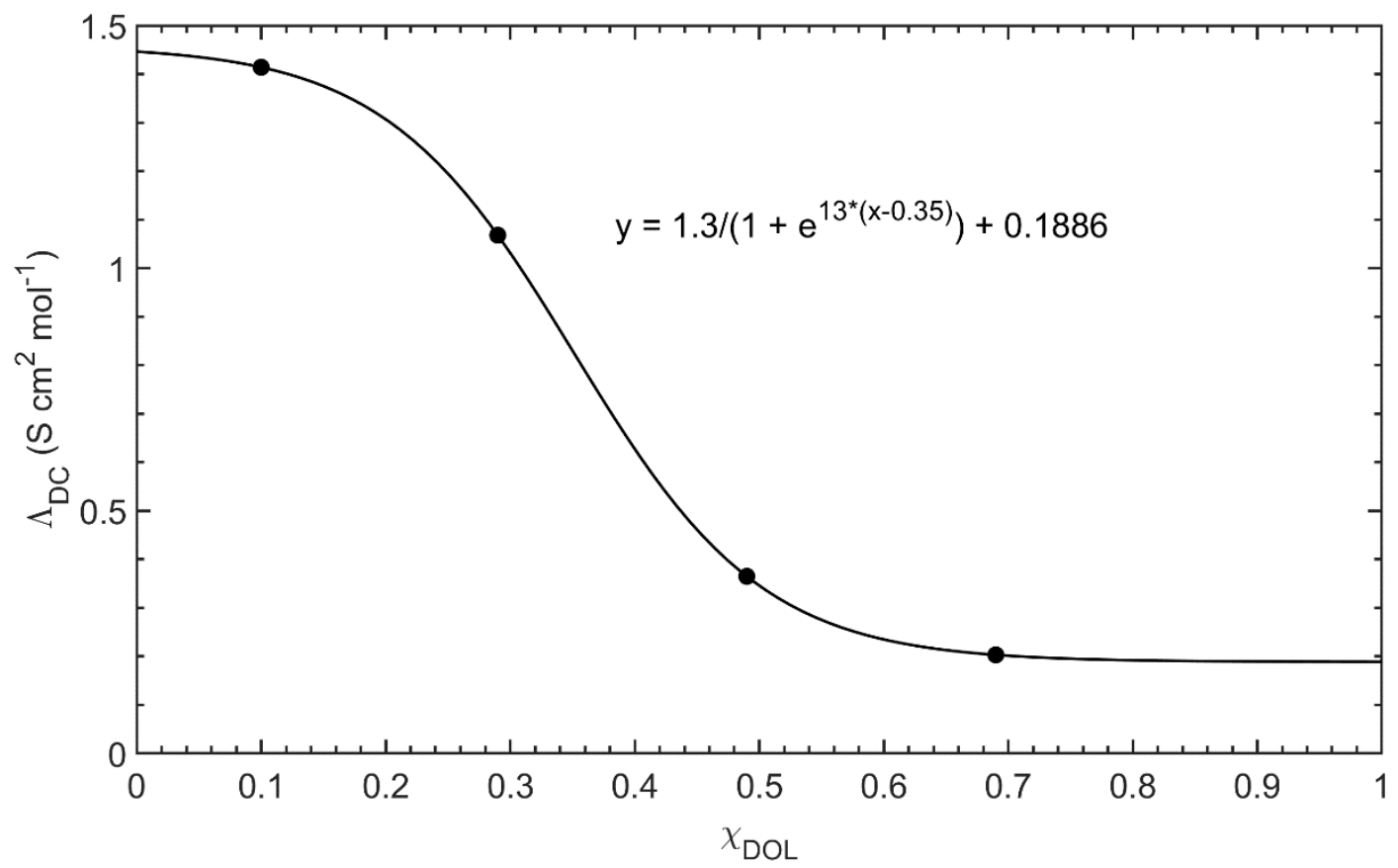


Figure S6: Power law exponent n vs. DOL fraction (showing 95\% confidence bounds), indicating the increasing dependence of viscosity on shear rate as DOL fraction increases.

Power Law Fit: $\eta=K(\dot{\gamma})^{n-1}$

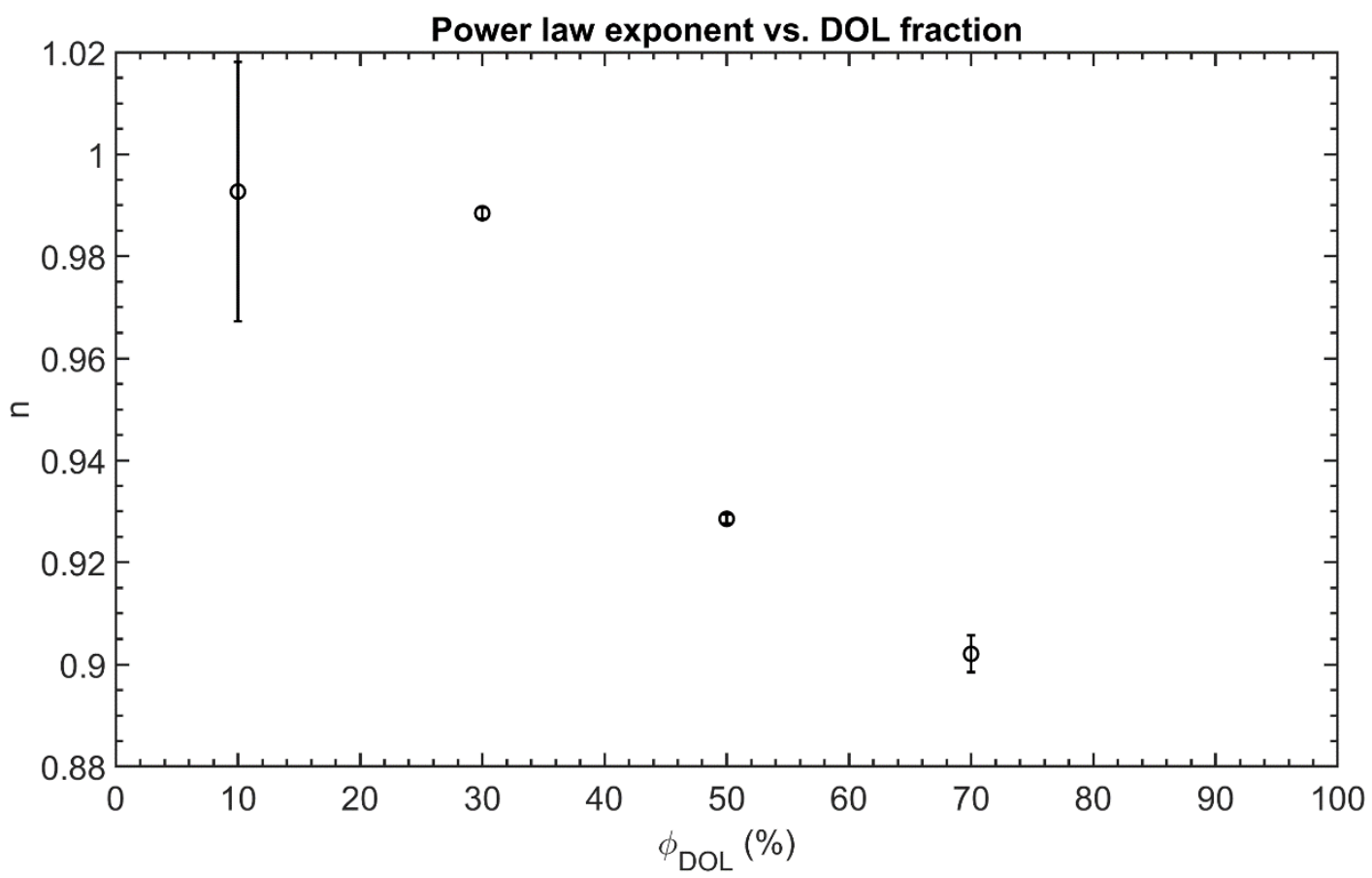

Table S3: Viscosity vs. shear rate power law fitting parameters for selected DOL fractions with $95 \%$ confidence bounds.

\begin{tabular}{|c|c|c|c|c|c|c|c|c|}
\hline & $\mathbf{n}$ & $\mathrm{nmin}$ & nmax & K & $\mathrm{Kmin}$ & Kmax & $R^{\wedge} 2$ & $\operatorname{adj} . R^{\wedge} \mathbf{2}$ \\
\hline $10 \%$ & 0.992687 & 0.96729 & 1.01808 & 0.00859 & 0.007852 & 0.009376 & 0.01993 & -0.03452 \\
\hline $30 \%$ & 0.98845 & 0.98741 & 0.9895 & 0.023768 & 0.023659 & 0.023933 & 0.9657 & 0.9639 \\
\hline $50 \%$ & 0.92854 & 0.92769 & 0.92939 & 0.779292 & 0.776247 & 0.782348 & 0.9993 & 0.9992 \\
\hline $70 \%$ & 0.90208 & 0.8985 & 0.90569 & 3.46657 & 3.411929 & 3.521275 & 0.9924 & 0.9921 \\
\hline
\end{tabular}


Figure S7: Phase Diagram DSC Traces. Scan rate $=10^{\circ} \mathrm{C} / \mathrm{min}$.

DSC Curves of EC/DOL, $1 \mathrm{M}$ LiPF6 at Selected DOL Volume Fractions

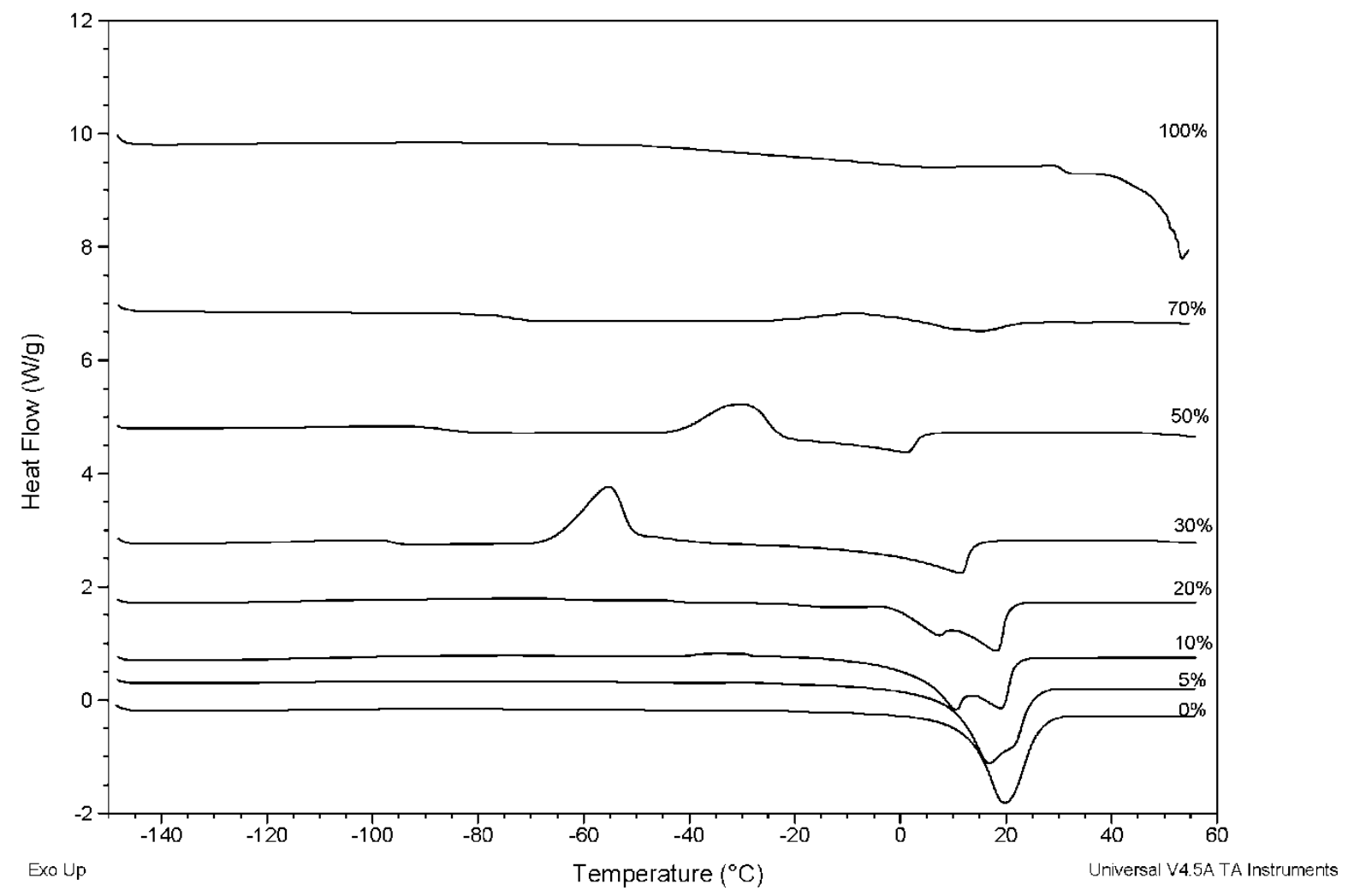


Figure S8: Lithium plating-stripping performance of EC/DOL/1 M LiPF 6 electrolytes measured in $\mathrm{Li}||$ stainless steel asymmetric cell at a rate of $1 \mathrm{~mA} / \mathrm{cm}^{2}$.
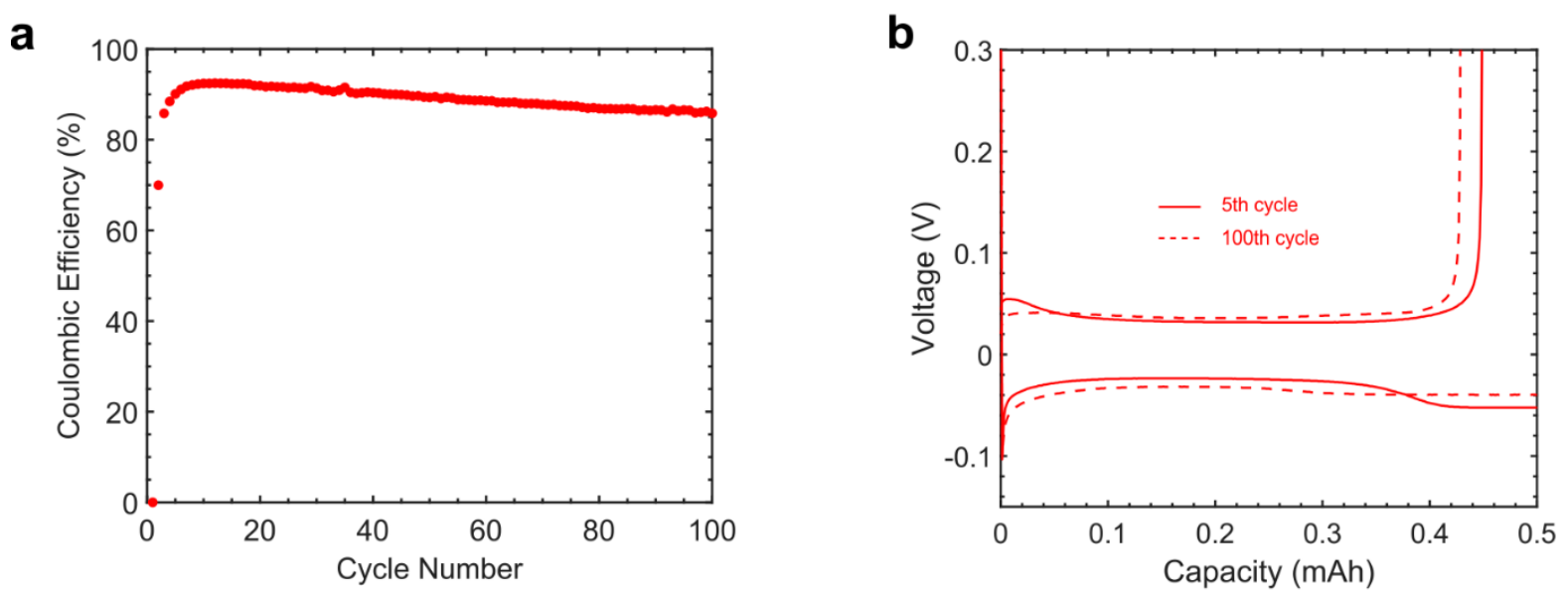

(a) Coulombic efficiencies and (b) corresponding voltage profiles as a function of cycle number. The DOL content in polymer electrolyte is $50 \%$.

Figure S9: Discharge/charge profiles for Li metal | NCM622 cells with EC/DOL/1 M LiPF 6 electrolytes.

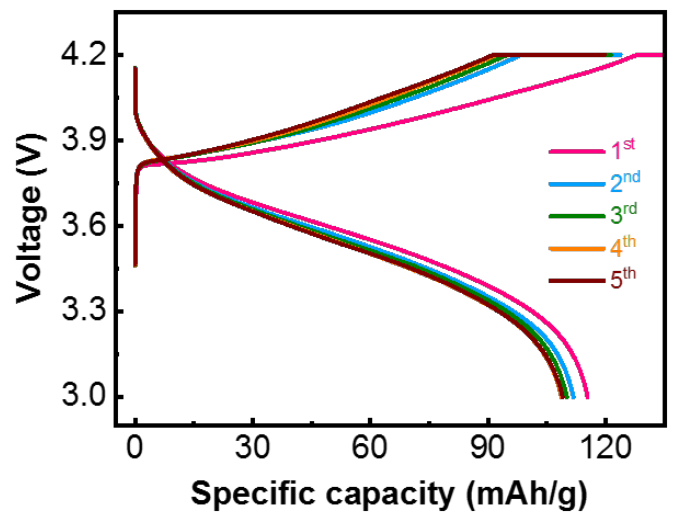

The DOL content in polymer electrolyte is $50 \%$. The cathode is nickel cobalt manganese oxide NCM622 $(60 \% \mathrm{Ni})$ with high areal capacity of $3 \mathrm{mAh} / \mathrm{cm}^{2}$. The cells are cycled at $0.2 \mathrm{C}$ in room temperature. 